\title{
DYNAMICS AND INTERACTIONS OF HIGH-REDSHIFT GALAXIES
}

\author{
M. NOGUCHI \\ Astronomical Institute, Tohoku University \\ Aoba, Sendai 980-77, Japan
}

\begin{abstract}
A large number of high redshift galaxies observed with the Hubble Space Telescope (HST) show anomalous morphology and photometric properties, which may be an indication of evolutionary process in young galaxies. We show here by means of numerical simulations that the copious interstellar gas existing in the disks of rapidly collapsing protogalaxies can bring about these peculiarities. Gravitational instability in a gas-rich disk leads to the formation of massive gas clumps with a typical mass of $10^{9} M_{\odot}$. These subgalactic clumps make disk galaxy evolution a dynamically energetic and chaotic process, and give a natural explanation for peculiar morphology of high redshift galaxies. Moreover, the present model provides a new picture on the causal relationship between the emergence of quasar activities and the dynamical evolution of host galaxies. The clumpdriven evolution model is also capable of explaining the correlations observed among present-day galaxies. Namely, the relative bulge dominance, existence of a thick disk, and a mass of the super-massive black hole situated at the galactic center should all be correlated positively. In contrast to their vigorous evolution in isolated state, primeval disk galaxies do not show any dramatic enhancement of activity or remarkable dynamical response in interaction with another galaxies.
\end{abstract}

\section{Gravitational Instability in Young Galactic Disks}

One of the most important characteristics of young galaxies is ample interstellar matter in their forming disks. The interstellar gas component is dynamically different from the stellar component in that the former is collisional and dissipative while the latter is collisionless. This difference manifests itself most remarkably in the presence of self-gravity. Therefore, 
the dominance of the gas in the disk galaxies at early cosmological epochs may bring about peculiarities in their structure and evolution.

In order to investigate the early phase of disk galaxy evolution, we simulated numerically the collapse of a rotating protogalaxy (Fig. 1, also Noguchi 1997). The protogalaxy was modeled as an ensemble of numerous gas clouds and dark matter particles distributed uniformly in a spherical volume. The masses of the gas component and the dark halo are identical. Inelastic collisions were introduced between gas clouds to simulate the dissipative nature of the gas, whereas the dark matter particles are assumed to move in a collisionless manner in the galaxy gravitational field. In the model depicted in Fig. 1, a uniform rotation was given so as to bring the system in a nearly centrifugal equilibrium initially. As seen in Fig. 1, the galaxy collapses nearly perpendicularly to the galactic plane within $\sim 1 \mathrm{Gyr}$.

The most remarkable feature in this model is that several distinctive clumps are formed as the disk is built up by the gas infall to the galactic plane. The typical mass of individual clumps measured from the simulation is $10^{9} M_{\odot}$. This is larger by three orders of magnitude than the masses of globular clusters and giant molecular clouds, which are most massive entities known in the present-day galaxies. These subgalactic clumps are created by the local gravitational instability of the mostly gaseous galactic disk in its early phase of formation. Star formation process is included in this simulation by converting gas clouds into stellar particles in accordance with a specified star formation law. The star formation rate reaches a maximum of $\sim 40 M_{\odot} y r^{-1}$ at the major epoch of disk formation $(t \sim 10)$. This is also the epoch when the clumpy structure is most prominent. The clumpy and sometimes asymmetric disk seen in this model is strongly reminiscent of the images of many galaxies at large redshift obtained by the $H S T$ (e.g., van den Bergh et al. 1996).

\section{Clump-driven Galaxy Evolution}

The subgalactic clumps play a key role in the evolution of disk galaxies. They experience strong dynamical friction owing to their large masses. Resulting accumulation of clumps to the galactic center makes a spheroidal bulge (Fig. 1, $t=30$ ). The bulge is not formed quickly in a single event as the protogalaxy collapses but is assembled gradually as individual clumps formed in the disk plane spiral into the galactic center. Fig. 2 shows the distribution of the age for the bulge stars at several epochs. At the final epoch indicated, $t=28$, the age spread is as large as $\sim 1 \mathrm{Gyr}$. The nature and evolution of bulges remain one of the important unresolved problems in the galactic astronomy (e.g., Rich 1996). Recent observations suggest that the bulge of the Milky Way galaxy has a large age range and shows 


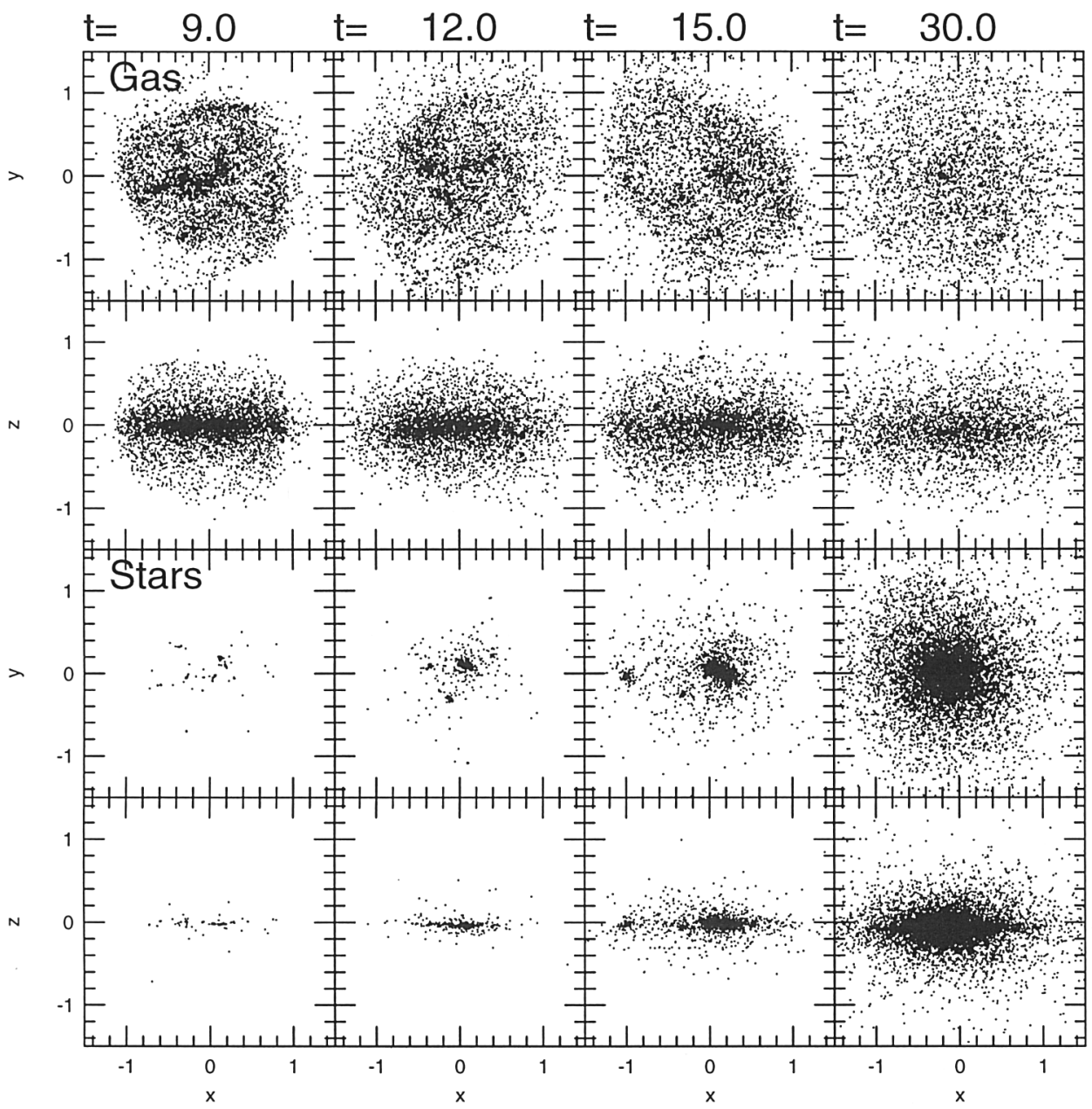

Figure 1. Morphological evolution of the disk galaxy model. The gas cloud particles and the stellar particles are projected onto the $x-y$ plane (i.e., the disk plane) and the $\mathrm{x}-\mathrm{z}$ plane. Time, $t$, is given in units of $\sim 10^{8} \mathrm{yr}$.

a correlation between abundance and kinematics of the constituent stars, which is difficult to explain by the conventional picture in which the bulge formation is a dynamically rapid process associated with the collapse of the protogalaxy. The clump-driven evolution model presented here may explain the enigmatic nature of the Milky Way bulge. The age distribution plotted in Fig. 2 indicates that the bulge sometimes contains a significant amount of young stars with ages less than $10^{8} \mathrm{yr}$. This is owing to the starburst caused by the falling of one of massive clumps into the bulge region. This behavior may explain the blue nucleated galaxies, which Schade et al. (1995) noted 


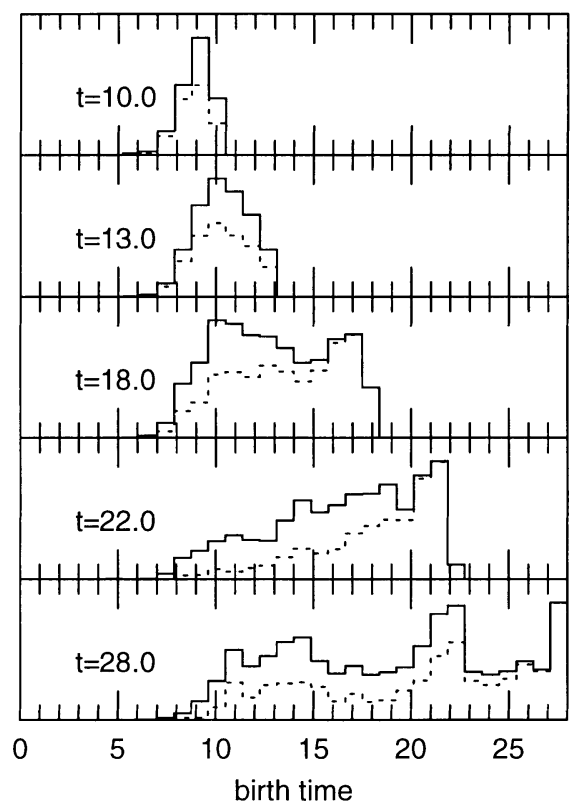

Figure 2. Distribution of the age for the stars contained in the bulge region. Each histogram shows, for the given epoch, the relative number of the stars which were born at the time indicated in the abscissa (unit time is $\sim 10^{8} \mathrm{yr}$ ). Solid lines are distributions for stars located within 0.1 length units of the bulge center, whereas the dotted lines for stars located within 0.025 .

among high redshift galaxy samples.

The clumpy disk has a tendency to acquire an exponential surface density distribution (Fig. 3), which is a universal feature of disk galaxies. This is understood naturally because a clumpy galactic disk in the present model can be viewed as a star-forming viscous disk (e.g., Saio \& Yoshii 1990). As the bulge grows, the rotation curve of the disk component gradually steepens and the radial zone having a constant rotational velocity widens, leading to a flat rotation curve, which is commonly observed in disk galaxies, especially of early morphological types (e.g., Rubin et al. 1985). While the clumps are orbiting in the disk plane, stars and gas clouds are scattered off them and acquire random motions because of large masses of individual clumps. The old stellar disk component thus shows a progressively large scaleheight as the time elapses and evolves into what is called a thick disk.

\section{Quasar Genesis}

The clump-driven evolution model provides a unique prediction on the emergence of active galactic nuclei (AGN) in evolving galaxies. The stan- 

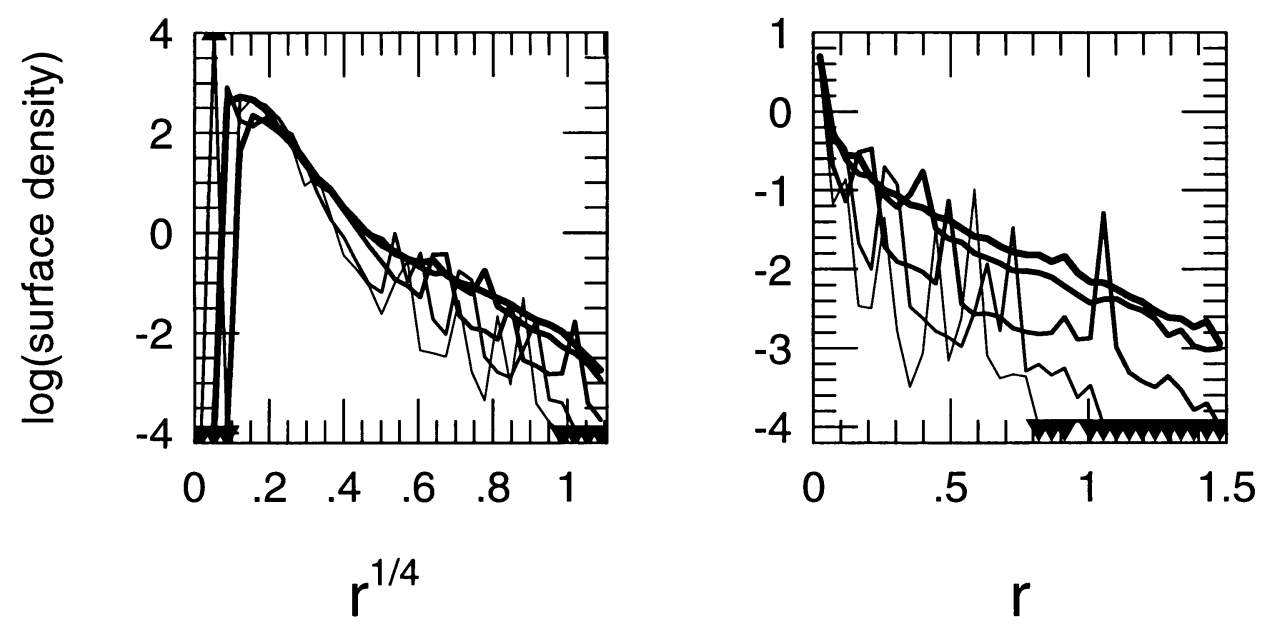

Figure 3. Growth of the bulge and disk components. Right panel plots the surface density distribution in the projection onto the $x-y$ plane for the stars as a function of the galactocentric distance. The progressively thicker lines correspond to the epochs, $t=$ $11,13,15,22$, and 29. Left panel is the same as the right panel except that the abscissa denotes $r^{1 / 4}$ to facilitate the comparison with the de Vaucouleurs' density law.

dard picture of AGN (including quasars) presumes, as their engines, a super-massive black hole located at the galactic center and fed by a gas infall from the surrounding accretion disk at a rate of $\sim 1 M_{\odot} y r^{-1}$. The mergers and accumulation of the heavy subgalactic clumps may provide an efficient way to cause the required inflow. As Fig. 4 indicates, each merger event between two clumps induces mass accretion onto those clumps with infall rate up to $\sim 20 M_{\odot} y r^{-1}$. Each merging pair may be regarded as a potential site of quasar activity. According to this scenario, there is no single well-defined epoch of quasar activity, and one single galaxy can be activated many times repeatedly and intermittently. Also, the active site need not coincide with the galaxy nucleus, because a clump merger can happen in the outskirts of the galactic disk. There is even a possibility of multiple quasars in principle. For example, a double quasar is formed when two pairs of colliding clumps happen to merge at nearly the same instant. The growth of the black hole population within a galaxy is hierarchical, from numerous small seed black holes which are embedded in clumps and orbiting in the disk, to successively larger ones via mergers, and finally to one (or a few) super-massive black hole(s) located in the nucleus. 


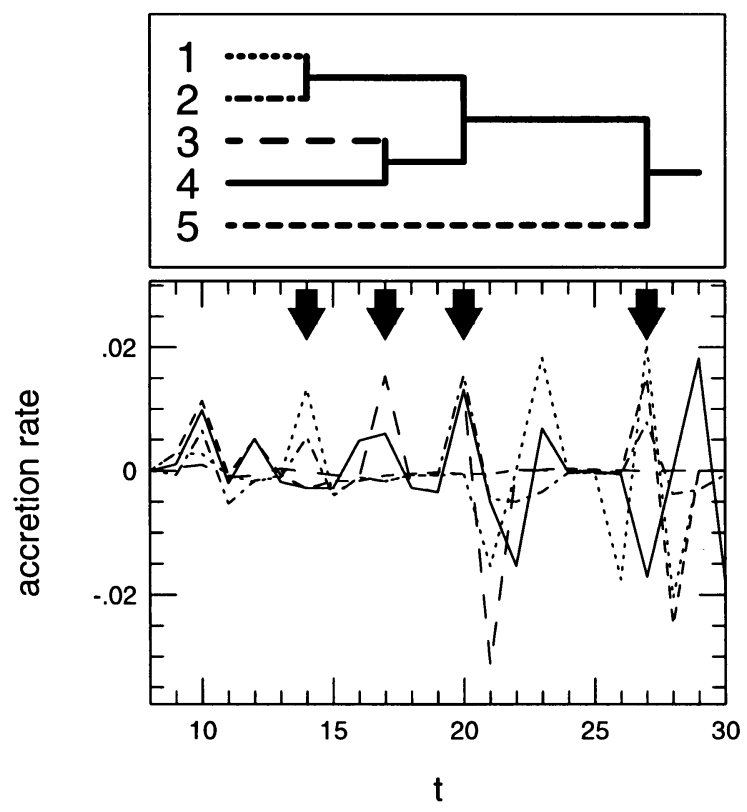

Figure 4. Merger history of the five selected clumps (upper panel) and the mass accretion onto individual clumps (lower panel). The same clump is indicated by the same line type in the upper and lower panels. Major accretion events associated with mergers between two clumps are indicated by arrows. A mass accretion of 0.01 corresponds to $\sim 20 M_{\odot} y r^{-1}$. Time, $t$, is in units of $\sim 10^{8} \mathrm{yr}$.

\section{Hubble Sequence}

What is the meaning of the Hubble morphological sequence in the framework of the clump-driven evolution model? If the disk formation is slowed down by some reason, the clumping of the disk is weakened because the disk contains less gas at its major formation epoch (see Noguchi 1996 for details). Thus all the clump-driven dynamical processes are subdued. The slow collapse model develops a smaller bulge component, has a thinner stellar disk, and a rotation curve rising outwards more gently. These features are all characteristic of late-type disk galaxies. The clumpy galaxy model presented here predicts that the prominence of the bulge should be correlated with the existence of a thick disk and the mass of the supermassive black hole in the galaxy nucleus (e.g., Kormendy \& Richstone 1995). Therefore, one possibility is that the Hubble sequence is largely that of disk formation time scale (Noguchi 1997). 


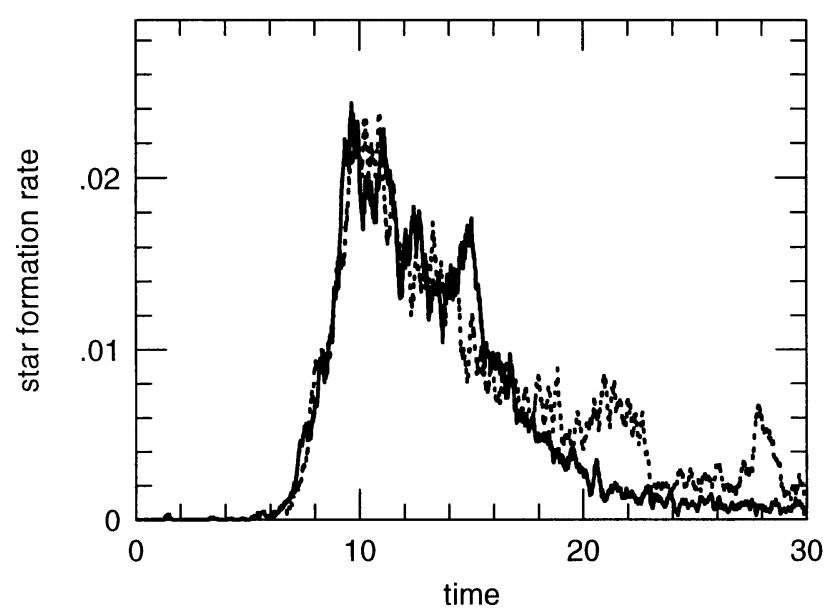

Figure 5. Star formation history of the perturbed galaxy model (solid) compared with that of the isolated evolution model (dotted). Star formation rate of 0.01 corresponds to $\sim 20 M_{\odot} y r^{-1}$.

\section{Interactions of Primeval Disk Galaxies}

In the nearby universe, many interacting and merging galaxies are observed to be sites of active star formation. Most of this starburst activity can be understood as a result of efficient gas flow to galactic centers induced either by tidally-created bars (Noguchi 1988) or merging process itself (e.g., Barnes \& Hernquist 1992). Numerical simulations have been carried out in which the clumpy galaxy model in its early evolution stage was made to collide with a perturbing galaxy (Noguchi 1998). No significant enhancement in star formation rate was observed (Fig. 5). The clumpy nature of the gas-rich disk prevents any coherent structure (such as a bar) from developing (Fig. 6). A completed galactic disk supported mainly by well-ordered rotational motion (such as seen in nearby disk galaxies) is necessary for a galaxy to respond dramatically in tidal interactions.

\section{References}

Barnes, J.E., and Hernquist, L. (1992), Ann. Rev. Astron. Astrophys. 30, 705-742

Kormendy, J., and Richstone, D. (1995), Ann. Rev. Astron. Astrophys. 33, 581-624

Noguchi, M. (1988), Astron. Astrophys. 203, 259-272

Noguchi, M. (1996), Astrophys. J. 469, 605-622

Noguchi, M. (1997), submitted

Noguchi, M. (1998), in preparation

Rich, R.M. (1996), in Unsolved Problems of the Milky Way (eds. Blitz, L. \& Teuben, P.) 403-410 (IAU Symp. No. 169, Kluwer Academic Publishers, Dordrecht)

Rubin, V.C., Burstein, D., Ford, W.K., and Thonnard, N. (1985), Astrophys. J. 289, 


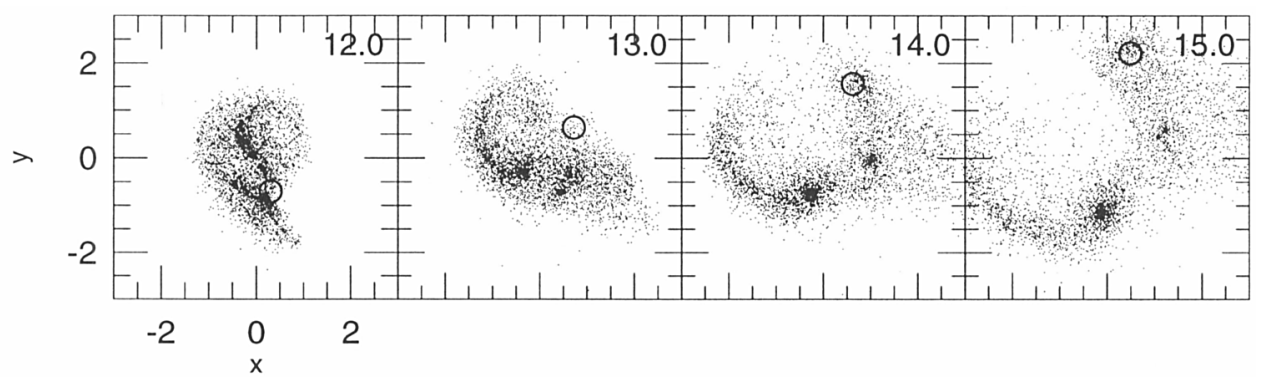

Figure 6. Morphological evolution of the perturbed galaxy model (to be compared with Fig. 1). Only gas clouds are shown. The circle indicates the position and effective radius of the Jaffe-type rigid perturber with a mass equal to that of the perturbed galaxy. Time in units of $\sim 10^{8} \mathrm{yr}$ is given in each panel.

81-104

Saio, H., and Yoshii, Y. (1990), Astrophys. J. 363, 40-49

Schade, D., Lilly, S.J., Crampton, D., Hammer, F., Le Fèvre, O., and Tresse, L. (1995), Astrophys. J. 451, L1-L4

van den Bergh, S., Abraham, R.G., Ellis, R.S., Tanvir, N.R., Santiago, B.X., and Glazebrook, K.G. (1996), Astron. J. 112, 359-368 